\title{
Are food composition databases ready to support food reformulation policies?
}

\author{
E. Combet ${ }^{1}$, S. Sharif ${ }^{1}$, C. Wratten ${ }^{1}$, G. Masset $^{2}$ and A. Vlassopoulos ${ }^{2}$ \\ ${ }^{1}$ School of Medicine, University of Glasgow, Glasgow, UK and ${ }^{2}$ Department of Public Health Nutrition, Nestlé \\ Research Center, Lausanne, Switzerland
}

Nutrient profiling (NP) is the classifying of foods according to their nutritional composition, for reasons related to promoting health. NP is therefore heavily dependent on the completeness of food composition tables. Potential applications of NP include food reformulation and the Nestlé Nutritional Profiling System (NNPS) has been designed specifically for that purpose ${ }^{(1)}$.

Here, we assessed whether national food composition databases- the UK McCance \& Widdowson, Chinese and Brazilian Food Composition databases and the US Food and Nutrient Database for Dietary Studies (FNDDS) - have the data completeness required to validate NP systems designed to guide food reformulation (for Energy, Total Fat, Saturated Fatty Acids, Sodium, Total Sugars and Added Sugars content). A sensitivity analysis was performed to assess the impact of using incomplete databases to validate NP systems using the NNPS as a case study (impact on the NNPS outcome Pass/Fail) (1). Three data handling scenarios were modelled 1. exclusion of incomplete food items, 2. imputation of nutrient content "high" or 3. imputation of nutrient content "low" (for McCance \& Widdowson only).

Granularity was assessed on the absolute number of food items amenable to reformulation available in each database. The FNDDS was the most detailed database, followed by the McCance \& Widdowson (Table 1). In most databases, one category represented the majority of food items with the remaining categories unequally populated. Data completeness was high for Energy, Total Fat and Sodium. SFA completeness was low in the Chinese database and Total/Added Sugars completeness was critical in the Brazilian and Chinese databases (Table 1). Data incompleteness was linked to high likelihood of introducing bias as the same approach (assume nutrient content high in case of missing value) can have opposing impact on the NP outcome (imputation scenario led to 3-fold increase in NNPS pass: NNPS fail ratio for "center of plate" foods vs 3-fold decrease in NNPS Pass: NNPS fail ratio for foods in the "Cakes, Cookies \& Desserts" category compared to the scenario rejecting foods with missing values)

Table 1. Estimates of granularity and completeness of food composition databases

\begin{tabular}{|c|c|c|c|c|c|}
\hline \multirow[b]{2}{*}{ Database } & \multirow[b]{2}{*}{$\%$ representation of main category } & \multirow{2}{*}{$\begin{array}{l}\text { No. of categories } \\
\text { with at least } 10 \text { items }\end{array}$} & \multicolumn{3}{|c|}{$\mathrm{N}$ missing and \% data completeness } \\
\hline & & & $\overline{\text { SFA }}$ & Added Sugars & Sodium \\
\hline $\mathrm{UK}(\mathrm{n}=1561)$ & $33.82 \%$ - Centre of plate & $24 / 35$ & $357(77 \cdot 1 \%)$ & $152(90 \cdot 3 \%)^{*}$ & $105(93 \cdot 3 \%)$ \\
\hline $\mathrm{US}(\mathrm{n}=3187)$ & $16.66 \%$ - Centre of plate & $28 / 35$ & $0(100 \%)$ & $0(100 \%)$ & $0(100 \%)$ \\
\hline China $(n=727)$ & $13.89 \%$ - Centre of plate & $18 / 35$ & $295(59.5 \%)$ & $727(100 \%)$ & $0(100 \%)$ \\
\hline Brazil $(\mathrm{n}=876)$ & $42.9 \%$ - Centre of plate & $13 / 35$ & $115(86.9 \%)$ & $739(15.7 \%)$ & $37(95.8 \%)$ \\
\hline
\end{tabular}

* Added sugar value calculated from total sugar, fructose, lactose, galactose, maltose values

Database quality based on granularity and data completeness for nutrient content is variable. The low level of information and detail available for foods belonging to less represented categories (i.e. savoury snacks) renders the databases a blunt tool to effectively segment the food supply and derive data-driven targets for reformulation and food policies.

Data completeness is very poor in some cases and even in more complete databases, missing nutrient information can be linked to the introduction of heterogeneous biases which can impact the performance of NP models.

1. Vlassopoulos A., et al. (2016). “A nutrient profiling system for the (re)formulation of a global food and beverage portfolio." Eur J Nutr 